\title{
Enabling transparent technologies for the development of highly granular flexible optical cross-connects
}

\author{
D. Klonidis' ${ }^{1}$, S. Sygletos ${ }^{2}$, D.M. Marom ${ }^{3}$, S. Fabbri' ${ }^{2}$ A. Ellis ${ }^{2}$, E. Pincemin ${ }^{4}$, C. Betoule, G. Thouenon ${ }^{4}$, D. \\ Hillerkuss $^{5}$, B. Baeuerle ${ }^{5}$, A. Josten ${ }^{5}$, J. Leuthold ${ }^{5}$, J. Zhao ${ }^{6}$, S. Ben-Ezra ${ }^{7}$, J.F. Ferran ${ }^{8}$, M. Angelou ${ }^{9}$, G. \\ Papastergiou $^{9}$, P. Zakynthinos ${ }^{1}$ and I. Tomkos ${ }^{1}$ \\ ${ }^{1}$ Athens Information Technology, 0.8 km Markopoulou Av., 19002, Paiania-Atika, Greece \\ ${ }^{2}$ Aston Institute of Photonic Technologies, Aston University, Birmingham, B4 7ET, UK \\ ${ }^{3}$ Applied Physics Department, Hebrew University, Jerusalem,91904, Israel \\ ${ }^{4}$ France Telecom, Orange Labs, 22307 Lannion Cedex, France \\ ${ }^{5}$ Institute of Electromagnetic Fields (IEF), ETH-Zurich, Zurich, Switzerland \\ ${ }^{6}$ Tyndall National Institute and University College Cork, Lee Maltings, Dyke Parade, Cork, Ireland \\ ${ }^{7}$ Finisar Israel, Nes-Ziona 74140, Israel \\ ${ }^{8}$ W-onesys, 78-84 Orient str., 08172, Sant Cugat del Vallès, Spain \\ ${ }^{9}$ Optronics Technologies S.A., 79-81 Thessalonikis str., 18346 Moschato, Athens, Greece \\ Tel: +30 210668 2700,Fax: +30 210668 2729, e-mail: dikl@ait.gr
}

\begin{abstract}
Flexible optical networking is identified today as the solution that offers smooth system upgradability towards $\mathrm{Tb} / \mathrm{s}$ capacities and optimized use of network resources. However, in order to fully exploit the potentials of flexible spectrum allocation and networking, the development of a flexible switching node is required capable to adaptively add, drop and switch tributaries with variable bandwidth characteristics from/to ultra-high capacity wavelength channels at the lowest switching granularity.

This paper presents the main concept and technology solutions envisioned by the EU funded project FOX-C, which targets the design, development and evaluation of the first functional system prototype of flexible adddrop and switching cross-connects. The key developments enable ultra-fine switching granularity at the optical subcarrier level, providing end-to-end routing of any tributary channel with flexible bandwidth down to 10Gb/s (or even lower) carried over wavelength superchannels, each with an aggregated capacity beyond $1 \mathrm{~Tb} / \mathrm{s}$.
\end{abstract}

Keywords: Flexible optical networks, super-channel switching, wavelength selective switch, all-optical interferometric add drop,

\section{INTRODUCTION}

Recent innovations in optical communication technologies achieved the introduction of advanced modulation formats and digital communication techniques enabling $100 \mathrm{~Gb} / \mathrm{s}$ transmission over long distances, while $400 \mathrm{~Gb} / \mathrm{s}$ and beyond prototypes are already available by major vendor (e.g. see Ciena Wavelogic ${ }^{\circledR}$, AlcatelLucent Photonic Service Engine ${ }^{\circledR}$ and Infinera DTN-X ${ }^{\circledR}$ solutions). Simultaneously, the traditional network and capacity planning solutions in optical networking that were initially based on the use of predetermined fixed capacity connections among network nodes, are replaced by dynamic and flexible solutions assisted by the introduction of ROADM technology. Therefore, the advances in a) high speed transmission technologies (e.g. 100Gb/s PM-QPSK and 400Gb/s PM-16QAM) and b) ROADM-based networking schemes, form the current state-of the art in optical communications that allows the transport of ultra-high capacity wavelength paths in an automated and remotely provisioned manner. Although the aforementioned advances solve individually the issues of high capacity per wavelength and dynamic path allocation, their combination is rather contradictory, due to the coarse granularity that is offered today (i.e. at the wavelength level only) in combination with the ultra-high data rate per wavelength. Still, a costly and complicated electronic-based procedure is required at each multiplexing point (node) to map lower rate tributaries into high capacity optical paths. Furthermore, optical networks today operate under added complexity and cost due to the poor network efficiency, since operators need the provisioning of connections that fulfil the highest network demands (over-provisioning).

Whilst the concept of spectrally efficient super-channels, first introduced in [1], combined with the more recent concepts of flexible optical networking [2], [3], appear to address the capacity on demand issue, they still rely on electronic aggregation functions at each node. Switching in core nodes can only be performed transparently at the super-channel level with traditional optical filtering solutions applicable in WDM systems. Any processing of the supper-channel contents (having typically overlapping spectra) requires first the termination (i.e. reception) of the whole super-channel in the node and in turn the electronic processing and switching of its contents. Also, even when switching at the super-channel level is assumed, spectral gaps are required to be inserted between neighboring super-channels in order to prevent cross-talk. In this case, the switching granularity is restricted to that of the super-channel level, and although in general the minimum super-channel bandwidth can be flexibly defined, the required insertion of a spectral gap between them reduces significantly the overall spectral efficiency. Significant research efforts [4]-[6] have targeted the use of add/drop filtering 
element with reduced spectral resolution in order to minimize the spectral gap width between super-channels. However, until recently, there were no filtering solutions with ultra-fine resolution close to $1 \mathrm{GHz}$ and more significantly, there were no solutions targeting the switching of super-channel contents with overlapping spectra directly in the optical domain.

The work performed within the EU funded FOX-C project (www.ict-fox-c.eu) investigates and develops solutions and enabling technologies that utilize the power of photonics to offer dynamic aggregation and switching in flexible optical nodes, directly at the super-channel content level (defined as sub-channel level). Such solutions can achieve ultra-low switching granularity resulting in significantly enhanced spectral efficiency and reduced energy consumption. Moreover the enhanced granularity offered by all-optical switching solutions enables the redefinition of the super-channel concept in terms of traffic allocation flexibility and the resulted increase in network spectral efficiency (or equally the optimized use of resources).

This paper presents and discusses the latest innovations in the development of switching elements with ultrafine resolution and the design and implementation of all-optical add/drop subsystems for sub-channels with overlapping spectra, stemming from the research efforts within the FOX-C project. The paper focuses then on the expected benefits and the new definitions for flexible optical networking that arise.

\section{Transparent switching elements, technologies and subsystems enabling flexible optical node designs}

The development of flexible optical nodes with increased granularity and spectral efficiency requires ideally the use of photonic elements able to provide mapping of low rate channels (coming from access or metro segments) into high data rate optical paths (in the backbone/core segment) in a transparent and flexible manner with reduced cost and energy consumption. Moreover, the support of the 'flexibility' concept defines tuneability, in terms of both bandwidth and wavelength allocation, in order to efficiently handle capacity changes due to traffic variations and optimize the core channel bandwidth utilization. Two types of switching subsystems are defined for the realization of the FOX-C node concept that can collaboratively enable the aforementioned requirements:

a. A tuneable and wideband ultra-fine filtering resolution switching element able to select and extract bandwidth flexible supper-channels out of a broad set of wavelength multiplexed super-channels transported in the optical links, while also separate its contents for further processing and even provide pre-shaping functions for the newly added channel spectra

b. An all-optical drop-erase-add subsystem capable to process specific contents of the super-channel without affecting the performance of the rest, even if they overlap in spectrum (OFDM case) or have zero guard bands between them (pure, i.e. guard-band free, Nyquist-WDM case).

These have been very recently presented in [7] and [8] respectively. Their key functions and characteristics are presented shortly in the following subsections.

\subsection{Flexible switching and high resolution adaptive filter elements}

The operating principle of a high resolution switching processor [9] is quite similar to that of wavelength selective switches. However, the bulk diffraction grating dispersive element is replaced by an engineered phase array designed to provide the optical resolution over a finite bandwidth. The spectral switching element is still an LCoS, now operating under much finer spectral granularity. A state-of-the-art phased array has been implemented in [7], where a high resolution AWG was designed to achieve $1 \mathrm{GHz}$ optical resolution metric and at most $200 \mathrm{MHz}$ spectral granularity. This first AWG prototype was designed with $50 \mathrm{GHz}$ FSR and was implemented in a silica on silicon platform. Since the length difference between the shortest and longest waveguides is $\sim 250 \mathrm{~mm}$ (to obtain the $1 \mathrm{GHz}$ resolution), the waveguides are folded three times within the PLC (see Figure $1 \mathrm{~b}$ ), resulting in a total size of $5 \times 2 \mathrm{~cm}$.
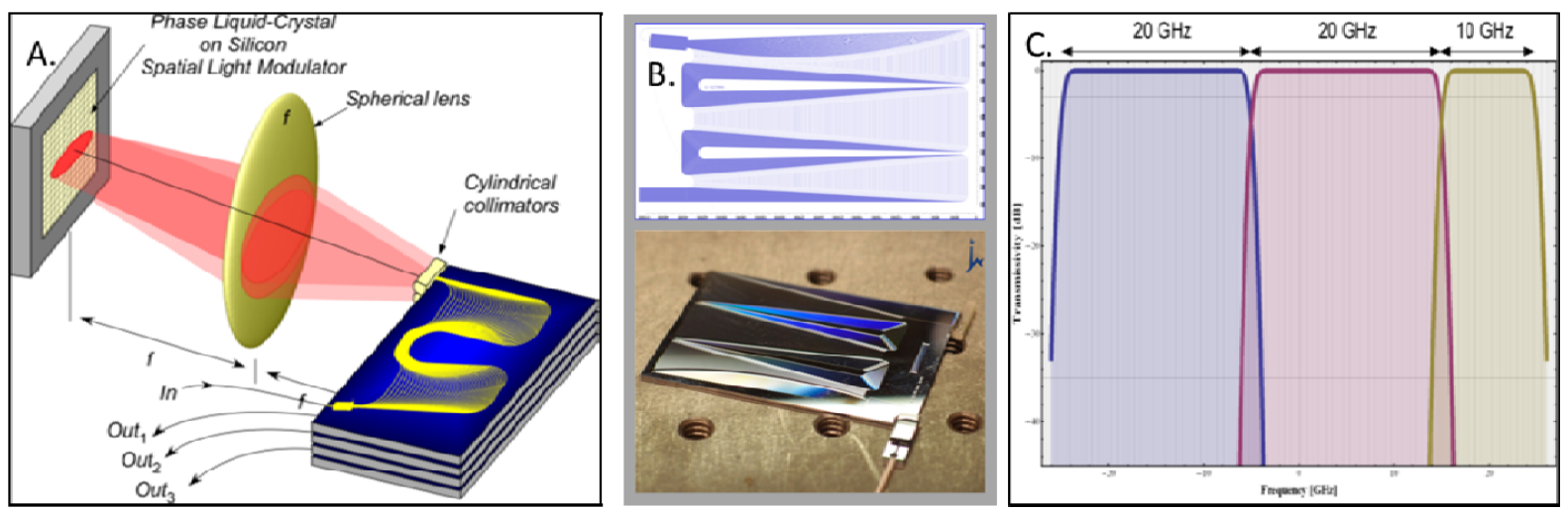

Figure 1. A) The high resolution filter, employing AWG for high resolution dispersion and an LCoS switching engine. B) The AWG design and implementation providing $<1 \mathrm{GHz}$ optical resolution and $200 \mathrm{MHz}$ spectral granularity C) Calculated fine spectral filter performance characteristics for three flexible sub-channels. 
The fine optical filter switching processor depicted in Figure 1a, can consist of a stack of AWG, each representing an input/output port, a free-space optical arrangement for dispersing the optical signal onto an LCoS SLM, and the LCoS SLM controlled by a computer that can assign spectral quanta as fine as the spectral granularity ( $<200 \mathrm{MHz}$ ) to subdivide the super-channel to finer tributaries. One fibre port (AWG) accepts a super-channel of any bandwidth up to the free-spectral range limit of the AWG (in this case 200GHz). The super-channel is then dispersed with a lens on the LCoS SLM at fine resolution. The LCoS selects the spectral bandwidth that will be routed to individual output AWG. These output AWG are assigned to a 'drop' side (can be one or more) and a 'through' destination, which will then be re-multiplexed with new 'add' data to form the new super-channel for upstream transmission. Since the optical resolution of the AWG is at record fidelity, the band transitions are extremely sharp, enabling the separation of the super-channel to tributaries with minimal guard bands.

In Figure 1c, the calculated finer spectral filter performance characteristics are shown, when variable bandwidth channels are deployed at spectral granularity of $200 \mathrm{MHz}$ (denoted by vertical grid lines) and an optical resolution of $1 \mathrm{GHz}$. This reveals the capability to extract even $10 \mathrm{GHz}$ channels with minimum spectral guard band of $2 \mathrm{GHz}$ between them, while achieving cross-talk suppression close to $20 \mathrm{~dB}$. The versatility of the AWG-based fine spectral filter is enabled by the LCoS processing, which is applied to the spectrally dispersed light (independently of the dispersion scheme). However, the challenge of this approach is the accuracy to which the AWG must conform. Briefly stated, all the AWG arms must radiate at the exact same relative phase while providing path length differences required to provide the spectral resolution value. A technique for correcting fabrication phase errors has been successfully demonstrated for the results obtained in [7].

\subsection{All-optical adaptive drop-erase-add subsystem}

Spectrally efficient superchannels rely typically on OFDM or Nyquist-WDM spectral contents [2], [3], [10] with overlapping spectra that are not able to be processed independently with all-optical filtering add/drop schemes due to severe cross-talk between the neighbouring multiplexed sub-channels. Recently, an optoelectronic interferometer structure has been proposed in [11] to facilitate sub-channel switching within OFDM bands, through coherent subtraction and addition of the sub-carriers. This scheme, however, deviates from the traditional concept of all-optical bypassing as it requires coherent detection and electronic de-multiplexing of the whole OFDM signal and the implementation of advanced digital pulse shaping to replicate the waveform of the sub-channel that needs to be removed from the super-channel. As a result, the scheme is not scalable to high superchannel capacities (e.g. > 400Gbit/s).

Based on the same interferometric processing principle, a novel approach has been developed and proposed in [8], by the researchers on FOX-C project, to perform add-drop and erase of the OFDM sub-channels in multicarrier OFDM super-channels directly in the optical domain without the use of optoelectronic conversion and digital signal processing. It is noted that the same scheme is also applicable to any format without modifications in the structure.

OFDM

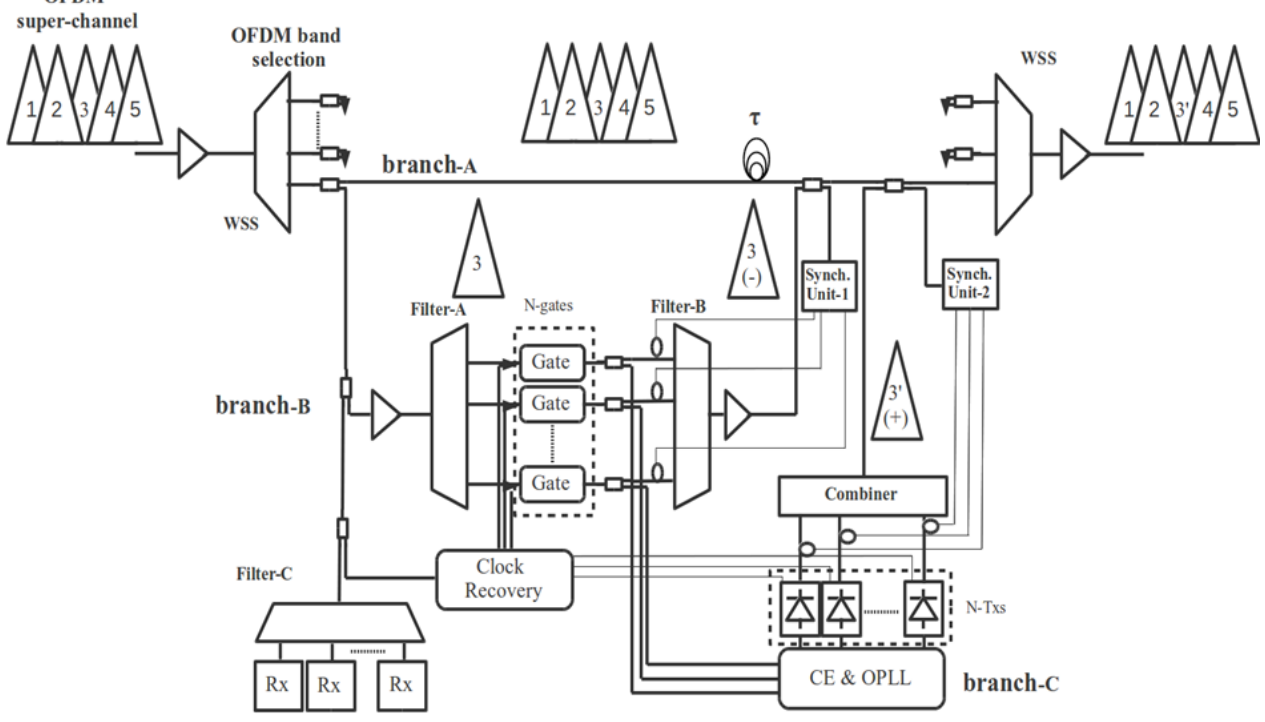

Figure 2. Reconfigurable optical add-drop multiplexer node (ROADM) for signals with overlapping spectra, consisting of all-optical signal regeneration and destructive interference for slot cleanout feature.

The proposed interferometric architecture is depicted in Figure 2. The architecture comprises an interferometric branch for performing the clearing of the signal components belonging to the Drop sub-channel (here channel 3). For perfect clearing to occur, several conditions have to be met, such as frequency and phase 
synchronization, and precise replication of the dropped sub-channel for complete destructive interference. This replication process requires two filtering functions: The first is a matched filter form to the dropped sub-channel. The matched filtering nulls out the interference contributions of the other sub-channel during a fraction of the symbol duration and can be implemented with the use of a high resolution filter shown in previous subsection. Next, an optical gating operation samples this time instant, generating a broadband pulse containing only the dropped signal photons (and their amplitude and phase information). This wideband short-pulse is subsequently shaped to the original form of dropped signal prior to the destructive interference event. The fine filtering operation serves to first demultiplex the optical sub-channel and subsequently (after sampling) shape the cleaned signal back to the original spectral/temporal form.

\section{Enabled flexible optical networking approach with low switching granularity}

The all-optical switching technologies presented in the previous sections allows the design of a new flexible optical networking approach able to provide switching of low rate tributaries (sub-channels) directly from multiple ultra-high capacity super-channel links. To date, in literature the term super-channel has been used to describe a signal that consists of multiple carriers that may originate from the same laser source [10] or individual laser sources [13], yet is perceived as a single entity. Regardless of the technology used to generate it, essentially it is referred to a high bit rate channel that is transmitted and received (in whole or in part [14]) as a continuous waveband signal formed by combining several low-rate subcarriers. In that sense, a super-channel does not necessarily have the capability to add/drop some of its subcarriers at an intermediate node along its route. Here, the adopted super-channel approach considers a dynamically adaptable waveband that is assigned coarsely on an end-to-end basis (according to slowly varying traffic demands between nodes), while it is capable to add/drop or even switch some of its contents. Thus, the term "sub-channel" is introduced to define the data that are carried by a single optical carrier within a super-channel. In this context a variable number of subchannels form the contents of the super-channel.

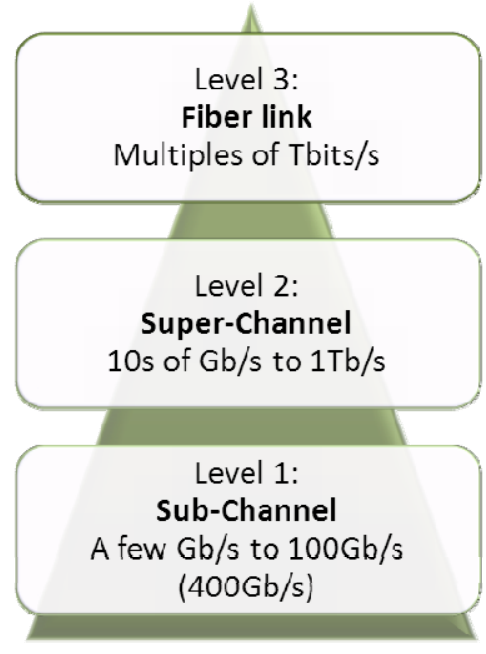

Switching granularity levels

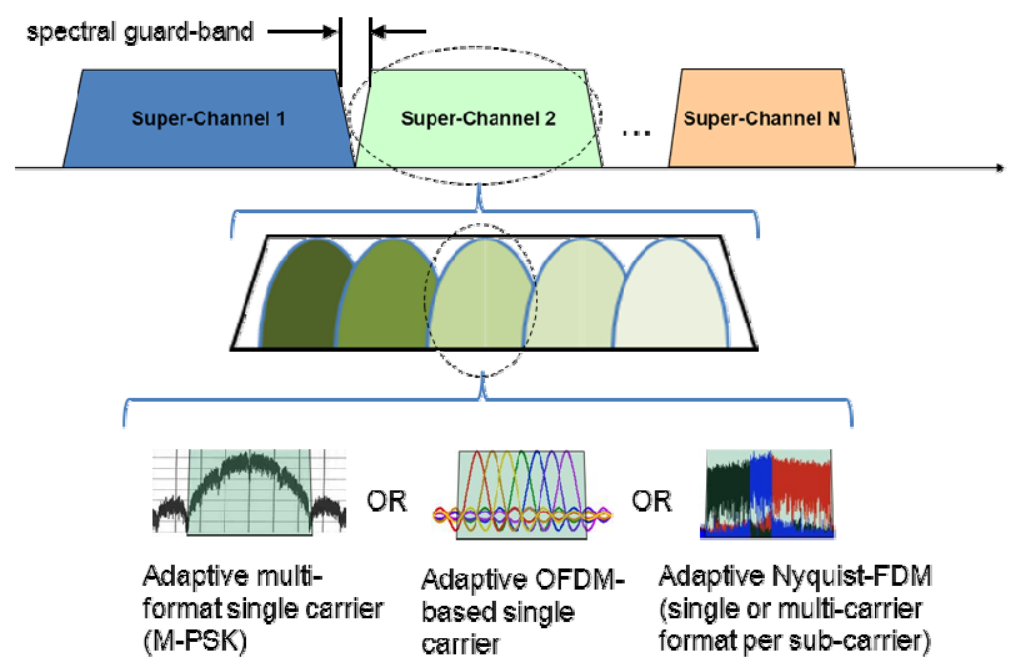

Figure 3. Definition of the three levels of granularity considered by the FOX-C project, including the superchannel structure and the multi-band adaptive transmission schemes.

Therefore, the new flexible optical networking scheme, promoted by the technologies developed in FOX-C project, defines three levels of data grooming and is depicted in Figure 3. According to this: low-rate tributary connections are being combined forming sub-channels (at level 1), which in turn are multiplexed to form spectral efficient and ultra-high capacity super-channels (at level 2) and finally multi-Terabit optical link connections (at level 3).

In turn, the signal transitions from one multiplexing level to another define two switching granularity levels per fibre. As a result the designed FOX-C flexible optical switching node architecture is based on two switching levels addressing a coarse switching resolution level (when super-channel are extracted and added from/to the WDM link) and a fine switching resolution level (when the contents of the super-channel, i.e. sub-channels are processed). This design is presented in the next sub-section.

\subsection{Flexible optical node design}

The key functional elements of the flexible node are presented in Figure 4 and described in the following paragraphs. At the fibre link level and from a number of high capacity (1Tb/s and beyond) super-channels in the core with flexible spectral characteristics, a WSS employing fine spectral selective elements (i.e. with a 
resolution below 6.25GHz) can select one super-channel that contains the sub-channel to be dropped locally or switched to another output fibre link. The rest of the super-channels continue directly to the output port (pass through function). Until this point the function of the node is similar to a typical WDM ROADM but with a finer adaptation to a variable spectral range and not to a fixed grid (e.g. 100GHz or 50GHz band) as in WDM. At the super-channel level, the contents of the selected super-channels are processed independently in parallel structures; (for simplicity, Figure 4 shows only the structure for one super-channel). The processes at this stage combine the technologies presented in section 2 of this paper. The channels that are to be dropped locally must be erased from the specific super-channel contents. Also the newly added signals must be coherently combined with the rest of the sub-channels forming the contents of the super-channel that will be later reinserted in the fibre link. This process is enabled by the all-optical interferometric structure. First, the dropped sub-channel(s) (may be one sub-channel or more supported by the same set-up with multiple stages in parallel) are selected with a fine resolution filter, gated, re-synchronised and re-shaped. The reshaped sub-channels will constructively erase the existing ones at the output of the interferometer. The newly added signals are written on top of synchronised and phase locked carriers of the dropped signals and combined with the rest of the sub-channels maintaining the orthogonality properties of the super-channel.

At the sub-channel level, the extracted sub-channels that are destined to the metro/access network segment are either expressed transparently to the lower segment of the system for processing at the access terminal nodes or coherently detected with the appropriate receivers and processed from common low rate switching elements (e.g. DXCs). Similar, at the add interface the encoded tributaries are either feed directly from the metro/access segments or the edge switches. It is noted that at this stage the dropped sub-channels can be switched back to the core network using a multi-port optical switch (e.g. MEMS switch). Such scheme allows the direct switching of sub-channels to other super-channels in the same optical link or even at different fibres. It can also implement selective regeneration at the sub-channel level. The latency is significantly minimised since the whole process can be realised purely in the optical layer.

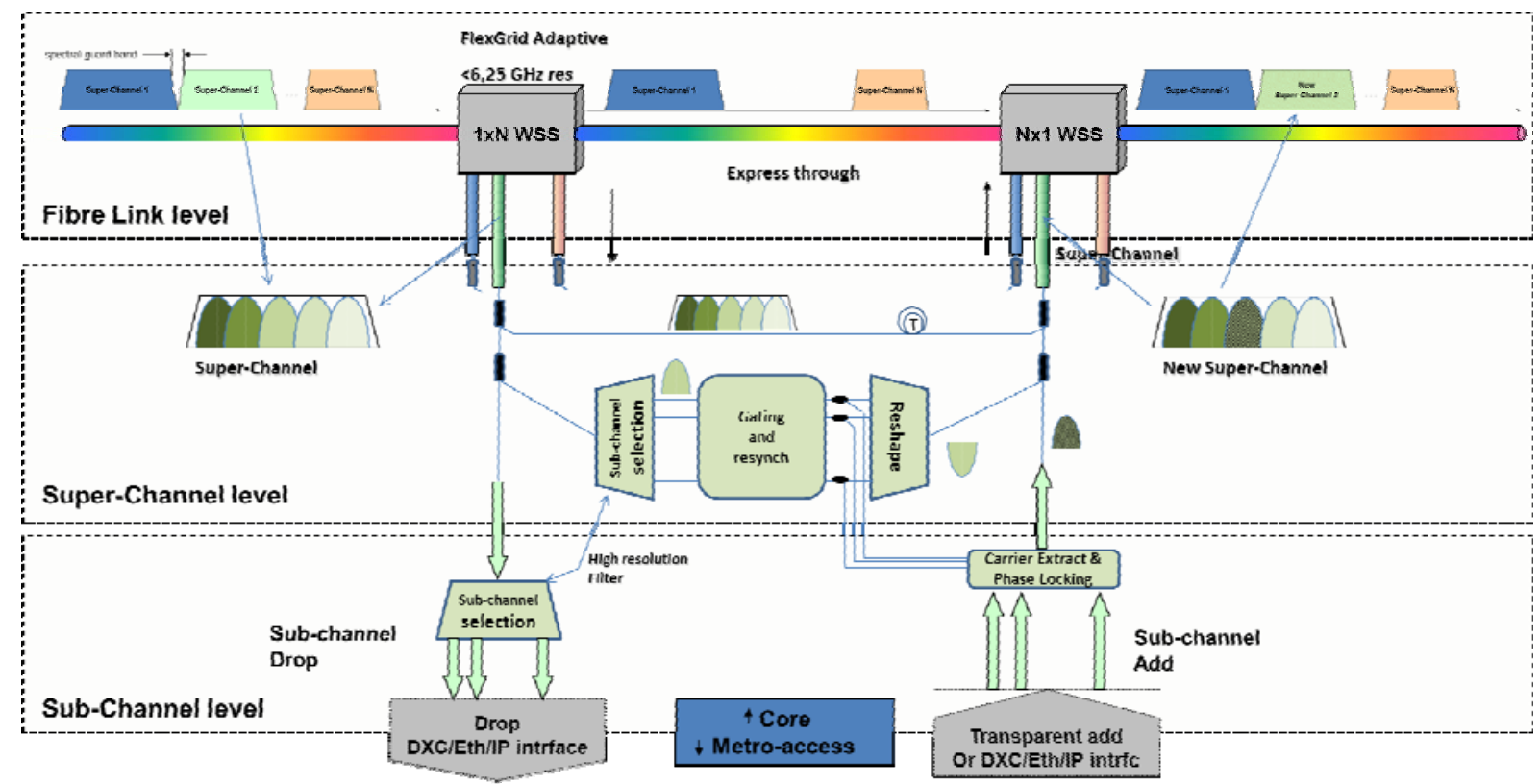

Figure 4. Functional elements of a flexible optical node with ultra-fine switching granularity following the three hierarchy levels defined by the FOX-C project and according to the enabled photonic technologies

\section{Conclusions}

Key photonic technologies that are developed within the EU funded FOX-C project enable the introduction of an advanced flexible optical networking concept, offering ultra-low switching granularity and fine grooming capabilities in the optical layer. These technologies focus on adaptive high resolution optical filters, achieving less than $1 \mathrm{GHz}$ optical resolution with at most $200 \mathrm{MHz}$ spectral granularity, and a novel all-optical interferometric add-drop and erase subsystem, providing sub-channel extraction and addition from/to the contents of a super-channel with overlapping spectra. Assisted by these enabling technologies, the new flexible optical networking concept allow low rate tributaries (defined at the minimum rate carried by one optical subcarrier inside a multi-carrier super-channel) to be extracted directly from ultra-high capacity super-channels in an all-optical manner, reducing therefore the overall switching latency and reducing significantly the energy consumption due mainly to the avoidance of any opto-electronic conversion and the appropriate DSP. 


\section{Acknowledgment}

The research leading to these results is carried out within FOX-C project and has received funding from the European Community's Seventh Framework Programme (FP7/2007-2013) under grant agreement n 318415”.

\section{REFERENCES}

[1] A.D. Ellis, F. Gunning, F. C G, "Spectral density enhancement using coherent WDM," Photonics Technology Letters, IEEE, vol.17, no.2, pp.504,506, Feb. 2005

[2] M. Jinno et al., "Spectrum-efficient and scalable elastic optical path network: architecture, benefits, and enabling technologies,” Communications Magazine, IEEE, vol. 47, no. 11, pp. 66-73, 2009.

[3] O. Gerstel, M. Jinno, A. Lord, and S. J. B. Yoo, "Elastic optical networking: a new dawn for the optical layer?” Communications Magazine, IEEE, vol. 50, no. 2, pp. s12-s20, 2012.

[4] S. Poole, S. Frisken, M. Roelens, and C. Cameron, "Bandwidth-flexible ROADMs as network elements," in proc. OFC/NFOEC 2011, paper OTuE1, March 2011.

[5] David Sinefeld, Shalva Ben-Ezra, and Dan M. Marom, "Nyquist-WDM filter shaping with a highresolution colorless photonic spectral processor,” Opt. Lett. 38, pp. 3268-3271, 2013.

[6] E. Pincemin et al., "Multi-band OFDM transmission with sub-band optical switching”, in proc. ECOC 2013, paper Th.2.A.1, 22-26 Sept. 2013

[7] Roy Rudnick, David Sinefeld, Ori Golani and Dan M. Marom, “One GHz Resolution Arrayed Waveguide Grating Filter with LCoS Phase Compensation”, in proc. OFC/NFOEC 2014, paper Th3F.7, March 2014.

[8] S. Sygletos et al., “A Novel Architecture for All-Optical Add-Drop Multiplexing of OFDM Signals”, in proc. ECOC 2014, Sept. 2014.

[9] David Sinefeld, Shalva Ben-Ezra, and Dan M. Marom, "Nyquist-WDM filter shaping with a highresolution colorless photonic spectral processor,” Opt. Lett. 38, pp. 3268-3271, 2013.

[10] X. Liu et al., "1.12-Tb/s 32-QAM-OFDM superchannel with 8.6-b/s/Hz intrachannel spectral efficiency and space-division multiplexing with 60-b/s/Hz aggregate spectral efficiency,” in Optical Communication (ECOC), 2011 37th European Conference and Exhibition on, 2011.

[11] Peter J. Winzer, “An Opto-Electronic Interferometer and Its Use in Subcarrier Add/Drop Multiplexing”, Lightwave Technology, Journal of, Vol. 31, No. 11, June 1, 2013, pp. 1775

[12] M. G. Taylor, “Coherent optical channel substitution,” U.S. Patent No. 8,050,564, Nov. 1, 2011.

[13] A. Klekamp, R. Dischler, and F. Buchali, "Transmission Reach of Optical-OFDM Superchannels with 10600 Gb/s for Transparent Bit-Rate Adaptive Networks,” in Proceedings of ECOC 2011, paper Tu.3.K.2.

[14] Shieh, William, "OFDM for Flexible High-Speed Optical Networks," Lightwave Technology, Journal of , vol.29, no.10, pp.1560,1577, May15, 2011 